\title{
ANALYSIS AND DESIGN OF SVC-BASED STABILIZER USING PARTICLE SWARM OPTIMIZATION TECHNIQUE
}

\author{
T. Fetouh", M. A. Abido ${ }^{* *}$, and A. A. Abou El-Ela* \\ "Department of Electrical Engineering, Faculty of Engineering, Minoufiya University, \\ Shebin El-Kom, Egypt \\ *** King Fahd University of Petroleum\& Minerals, Dhahran, Saudi Arabia
}

\begin{abstract}
This paper evaluates the performance and effect of the static VAR compensator (SVC) on the system stability enhancement. In addition, the paper presents a proposed method to coordinate between SVC-based stabilizer and power system stabilizer (PSS) through an optimization process for the system stability problem that employs a variety of single or multi-objective functions. These objective functions are based on the nonlinear model of the system. Particle Swarm Optimization (PSO) technique is applied to search for the optimal SVC and PSS controller parameters. The proposed method leads to more flexibility to the system by involving all the controller parameters in the optimization process and using different objective functions. The system response is analyzed with utilizing the PSS only and compared with utilizing the SVC coordinated with the PSS. The simulation results confirm that coordination between the PSS and the SVC as well as adding more flexibility to the system improves the system response.

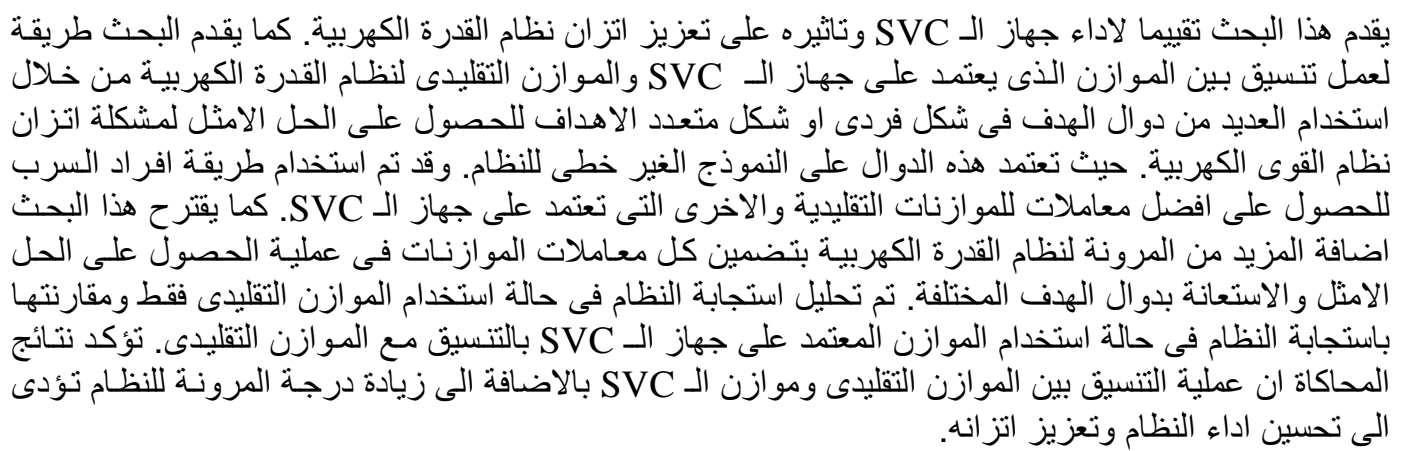

Keywords: Static VAR Compensator, Particle Swarm Optimization, and System Stability

\section{INTRODUCTION}

Damping of power system oscillations is very important for the system secure operation. Besides the PSS, the Flexible AC Transmission System (FACTS) devices are also applied to enhance system stability. Using only conventional PSS may not provide sufficient damping for system oscillations. In these cases, FACTS-based power oscillation damping controllers are effective solutions [1-3]. However, uncoordinated control of FACTS devices and PSS may cause destabilizing interactions. To improve overall system performance, studies should be made on the coordination between PSS and FACTS controllers [4-5].

Among the different types of FACTS devices, SVC is widely used in many electricity utilities over the world and attained great interest from the researcher [5-11]. In its simplest form, the SVC consists of a Thyristor Controlled Reactor (TCR) in parallel with a bank of capacitors [12-13]. From an operational point of view, the SVC behaves like a shunt-connected variable reactance, which either generates or absorbs reactive power in order to regulate the voltage magnitude at the point of connection to the AC network. It is used extensively to provide fast reactive power and voltage regulation support. The firing angle control of the thyristor enables the SVC to have almost instantaneous speed of response. In addition to the primary function of SVC, enhancement of system stability can be assigned to SVC as a supplementary function [5].

The literature addresses different methods to find the optimal location of SVC device [7-11]. The basic factors in these methods are the linear model of the system, modal analysis, and voltage stability analysis. The linearized models for power systems including SVC and other FACTS devices are usually used as a base for the optimization process $[1,4-5,7-$ 8]. 
Optimization techniques inspired by observing natural phenomenon have attracted the interest of many researchers. These techniques approved their ability to solve complex computational problem. Particle swarm optimization technique (PSO) [1415], can be used to find the optimal setting of the controller parameters of both PSS and SVC. The linearization effort can be saved and no approximations are needed to handle the controller design problem.

Usually, some controller parameters are predefined and kept constant during the optimization process [3, 4-5]. However, including these parameters in the optimization process will lead to more flexibility in the system operation.

Single Machine Infinite Bus (SMIB) system is used to analyze and design the PSS and SVC controllers. The two controllers are designed individually and compared with the coordinated controllers.

\section{POWER SYSTEM MODEL}

The single machine infinite bus system (SMIB) is used to extensive study of stability enhancement as shown in Fig. 1. The generator is equipped with PSS while, the SVC is placed at the mid-point of the transmission line.

\subsection{Generator Model}

The generator is represented by the third-order model, which is described by the following electromechanical swing and the generator internal voltage equations:

$$
\begin{aligned}
& \frac{d \omega}{d t}=\frac{1}{M}\left[P_{m}-P_{e}-D(\omega-1)\right] \\
& \frac{d \delta}{d t}=\omega_{b}(\omega-1) \\
& \frac{d E_{q}^{\prime}}{d t}=\frac{1}{T_{d o}^{\prime}}\left[E_{f d}-E_{q}^{\prime}-\left(x_{d}-x_{d}^{\prime}\right) \cdot i_{d}\right]
\end{aligned}
$$

where,

$P_{m}$ and $P_{e}$ are the input and output powers of the generator, respectively,

$M$ and $D$ are the inertia constant and damping coefficient, respectively,

$\delta$ and $\omega$ are the rotor angle and speed, respectively,

$E_{q}^{\prime}$ and $E_{f d}$ are the generator internal voltage and the field voltage, respectively.

$T_{d o}^{\prime} \quad$ is the open circuit time constant of the field circuit.

$x_{d}$ and $x_{d}^{\prime}$ are the $d$-axis reactance and $d$-axis transient reactance of the generator, respectively.

The generator output power can be expressed in terms of the $d$-axis and q-axis components of the armature current $(i)$, and the terminal voltage $\left(V_{t}\right)$, as follows:

$$
P_{e}=v_{d} \cdot i_{d}+v_{q} \cdot i_{q}
$$

The terminal voltage $V_{t}$ can be expressed as:

$$
\begin{aligned}
& V_{t}=\sqrt{v_{d}^{2}+v_{q}^{2}} \\
& v_{d}=x_{q} \cdot i_{q} \\
& v_{q}=E_{q}^{\prime}-x_{d}^{\prime} \cdot i_{d}
\end{aligned}
$$

where,

$$
X_{q} \text { is the } q \text {-axis reactance of the generator. }
$$

\subsection{Exciter and PSS Model}

The IEEE Type-ST1 [5], is considered as the excitation system. It is equipped with a conventional lead-lag PSS in the feedback loop to generate a stabilizing signal $U_{P S S}$, as shown in Fig. 2. The exciter can be described by the following equation:

$\frac{d E_{f d}}{d t}=\frac{1}{T_{A}}\left[K_{A}\left(V_{r e f}-V_{t}+U_{p s s}\right)-E_{f d}\right]$

where,

$K_{A}$ and $T_{A}$ are the gain and time constant of the excitation system, respectively.

$V_{\text {ref }} \quad$ is the reference voltage.

\subsection{SVC-based Stabilizer Model}

The block diagram shown in Fig. 3 represents the SVC with lead-lag controller. A conventional leadlag controller is installed in the feedback loop to generate the SVC stabilizing signal. The SVC is represented by a susceptance $\left(B_{\mathrm{SVC}}\right)$, which is governed by the following equation:

$\frac{d B_{s v c}}{d t}=\frac{1}{T_{S}}\left[K_{S}\left(B_{r e f}+U_{s v c}\right)-B_{s v c}\right]$

where,

$K_{S}$ and $T_{S}$ are the gain and time constant of the SVC, respectively.

$B_{\text {ref }} \quad$ is the reference susceptance of the SVC.

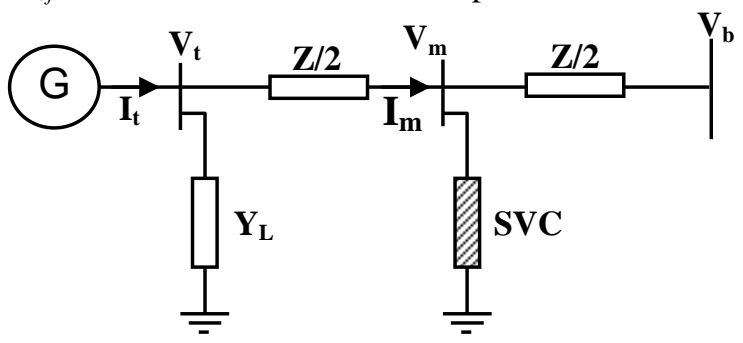

Fig. 1. Single Machine Infinite Bus System (SMIB)

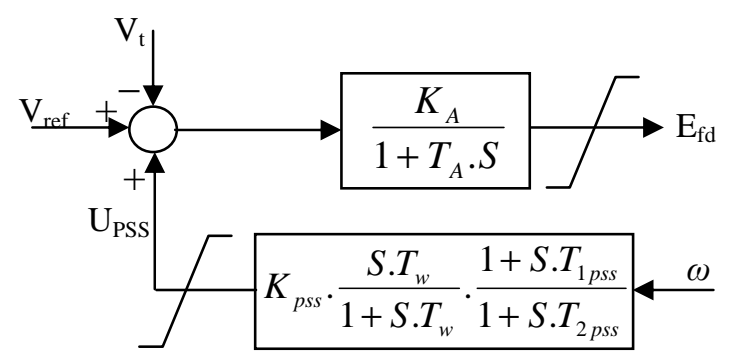

Fig. 2. IEEE Type-ST1 Excitation system with PSS 


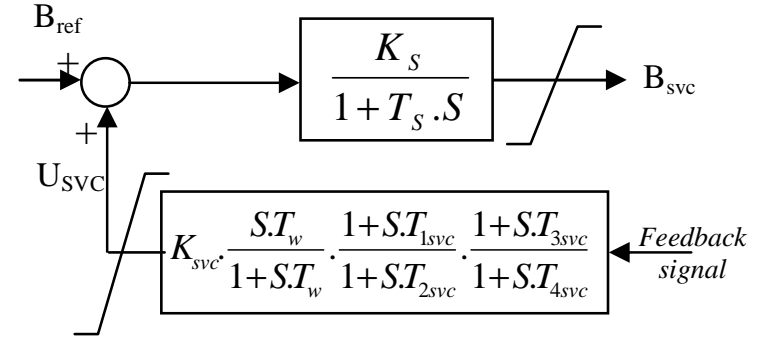

Fig. 3. SVC with lead-lag controller

\section{PROBLEM FORMULATION}

The parameters of PSS and SVC-based stabilizers are determined, through the optimization process, to improve the system response. Phillips-Heffron linearized model around an operating point is usually employed for design purposes [6-7]. However, the modern optimization techniques approved their ability to solve the nonlinear optimization problems. Therefore, nonlinear model is used for the design process.

\subsection{Particle Swarm Optimization Technique}

The PSO is a population based stochastic optimization technique developed by Kennedy and Eberhart in 1995 [14-15], inspired by social behavior of bird flocking or fish schooling. The system is initialized with a population of random solutions and searches for optima by updating generations. PSO provides a population-based search procedure in which chromosomes called particles change their positions with time. In a PSO system, particles fly around in a multidimensional search space. During flight, each particle adjusts its position according to its own experience, and the experience of neighboring particles, making use of the best position encountered by itself and its neighbors. The swarm direction of a particle is defined by the set of particles neighboring the particle and its history experience.

In Searching procedures by PSO the particles are flown through the problem space by following the current optimum particles. Each particle keeps track of its coordinates, which are associated with the best solution that it has achieved so far. This solution is called personal best (pbest) and it is kept in the particle memory as the best position on the feasible search space. The best of value obtained so far by any particle in the group is commonly called the global best (gbest).

The basic concept behind the PSO technique consists of changing the velocity of each particle toward its pbest and the gbest positions at each iteration. The velocity and position of each particle can be modified according to the distance between its current position and pbest, and the distance between its current position and gbest. Using Equation (10), a certain velocity that gradually gets close to pbests and gbest can be calculated. The current position can be modified using Equation (11).

$$
\begin{aligned}
& v_{i d}^{k+1}=w \cdot v_{i d}^{k}+c_{1} \cdot r_{1} \cdot\left(\text { pbest }_{i d}-x_{i d}^{k}\right) \\
& +c_{2} \cdot r_{2} \cdot\left(\text { gbest }-x_{i d}^{k}\right) \\
& x_{i d}^{k+1}=x_{i d}^{k}+v_{i d}^{k+1} \\
& i=1,2, \ldots ., n \quad d=1,2, \ldots ., m
\end{aligned}
$$

Where,

$n \quad$ is the number of particles in a population,

$m$ is the number of members in a particle,

$v_{i}^{k} \quad$ is the current velocity of particle $i$ at iteration $k$,

$v_{i}^{k+1}$ is the modified velocity of particle $i$ at iteration $k+1$

$x_{i}^{k} \quad$ is the current position of particle $i$ at iteration $k$,

$x_{i}^{k+1} \quad$ is the modified position of particle $i$ at iteration $k+1$

$r_{1}, r_{2}$ are the random number between 0 and 1 ,

pbest $_{i}$ is the personal best of particle $i$,

gbest is the global best of the population,

$c_{1}, c_{2}$ are the acceleration constants.

$w \quad$ is the inertia weight parameter

The inertia weight parameter $(w)$ often decreases linearly from $w_{\max }$ to $w_{\min }$ during a run. In general, the inertia weight $(w)$ is set according to the following equation:

$w=w_{\max }-\frac{w_{\max }-w_{\min }}{k_{\max }} * k$

Where $k_{\max }$ is the maximum number of iterations, and $k$ is the current number of iterations.

The modifications of searching points continue until the maximum number of iterations is reached or the solution has no changes for a specific number of iterations.

\subsection{Objective Function}

The objective function in case of linear model-based optimization problem is limited to maximizing the damping ratio. However, nonlinear model-based optimization problem has a variety of objective functions that can be treated as single or multiobjective optimization problem. The following nonlinear model-based objective functions can be defined for different design cases, all of these objective function need to be minimized.

$$
\begin{aligned}
& \text { Min, } J_{1}=\int_{\text {Finst }}^{\text {Tsim }}|\omega-1| . d t \\
& \text { Min, } J_{2}=\int_{\text {Finst }}^{\text {Tsim }}\left|\delta-\delta_{0}\right| . d t
\end{aligned}
$$


$\operatorname{Min}, J_{3}=w_{1} . J_{1}+w_{2} . J_{2}$

Where,

$\begin{array}{ll}F_{\text {inst }} & \text { is the fault instant } \\ T_{\text {sim }} & \text { is the simulation time } \\ w_{1}, w_{2} & \text { are the weighting factors }\end{array}$

However, the following linear model-based objective function needs to be maximized through the optimization process:

$\operatorname{Max}, J_{4}=\zeta=\frac{-\sigma}{\sqrt{\sigma^{2}+\omega^{2}}}$

\subsection{Optimization Problem}

The aim of the optimization problem is to minimize the objective function while satisfying all the system constraints. Usually, some of controller parameters, $\left(T_{2 p s s}, T_{2 s v c}, T_{4 s v c}\right)$, are predefined and kept constant during the optimization process. However, including these parameters in the optimization process will lead to more flexibility in the system operation. The parameters of the PSS are optimized in coordinate with the SVC controller parameters. Therefore, the optimization problem can be formulated as follows:

\section{Minimize $J_{1}$, or $J_{2}$, or $J_{3}$}

Or Maximize $\mathrm{J}_{4}$

Subject to:

$$
\begin{aligned}
& T_{\text {lpss-min }} \leq T_{\text {lpss }} \leq T_{\text {lpss-max }} \\
& T_{2 p s s-\min } \leq T_{2 p s s} \leq T_{2 p s s-\max } \\
& K_{p s s-\min } \leq K_{p s s} \leq K_{p s s-\max } \\
& T_{1 s v c-\min } \leq T_{1 s v c} \leq T_{1 s v c-\max } \\
& T_{2 s v c-\min } \leq T_{2 s v c} \leq T_{2 s v c-\max } \\
& T_{3 s v c-\min } \leq T_{3 s v c} \leq T_{3 s v c-\max } \\
& T_{4 s v c-\min } \leq T_{4 s v c} \leq T_{4 s v c-\max } \\
& K_{s v c-\min } \leq K_{s v c} \leq K_{s v c-\max }
\end{aligned}
$$

\section{RESULTS AND DISCUSSION}

In this study, the SVC-based stabilizer parameters have been optimized and coordinated with those of PSS using PSO technique. The nonlinear time response for a three-phase short circuit is tested at different controller types is shown in Fig. 4. It is clear that, using the SVC-based stabilizer coordinated with PSS improves the system response. The first overshoot is improved, and the system reaches to its steady state quickly. However, the individual use of the SVC may lead to negative damping to the system.

Figure 5, shows the effect of involving all the controllers parameters in the optimization process. More flexibility is added to the system and the system response is improved rather than using fixed values for some parameters. The optimal setting of the controller parameters in the two cases is shown in Table 1.
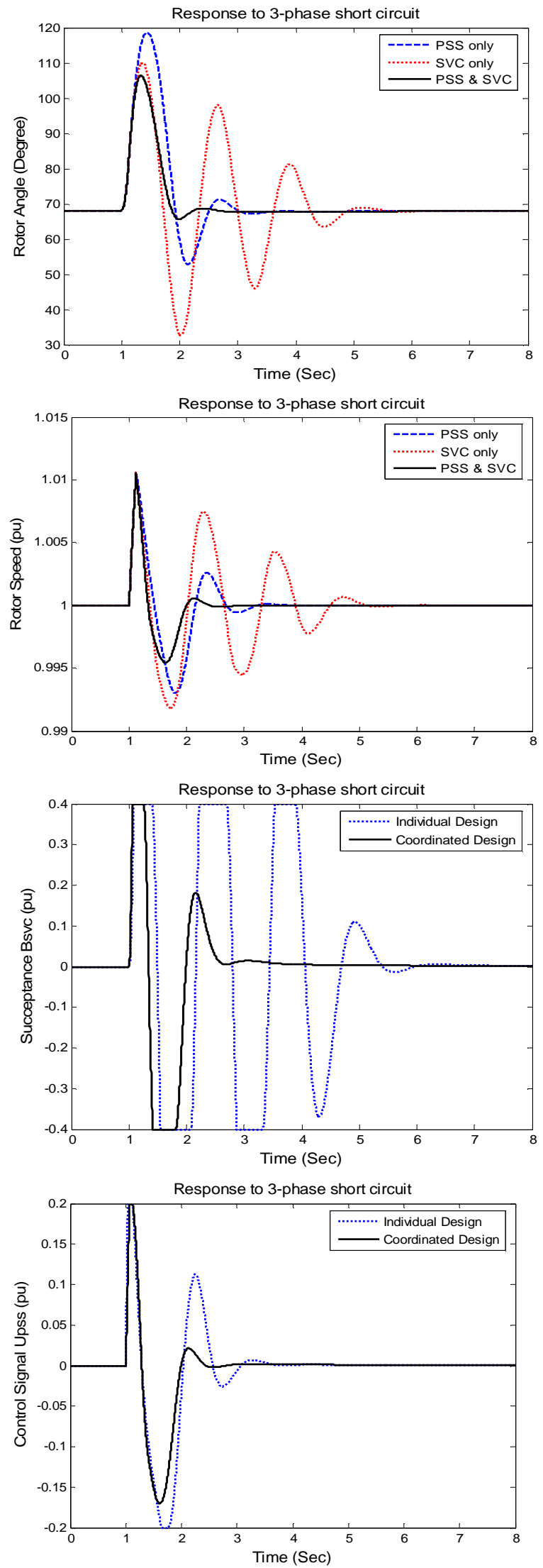

Fig. 4. System response under different controller types 
Figure 6, shows a comparison between the system response when applying different objective functions in the optimization problem. The nonlinear modelbased optimized parameters are applied to the coordinated controllers and the system response is compared with that of linear model-based parameters as shown in Fig. 6. It is clear that the non-linear model-based system response is quite better than the linear model.
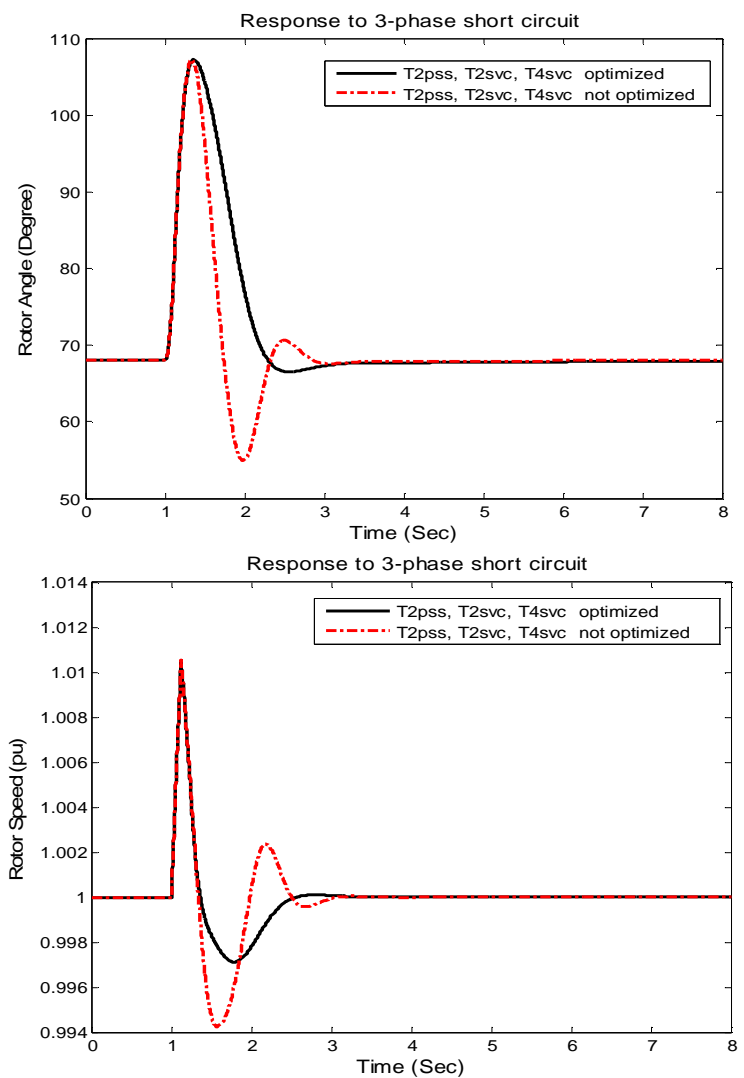

Fig. 5. System response for different cases of optimized parameters

Table 1, Optimal setting of coordinated PSS and SVC Controller parameters

\begin{tabular}{ccc}
\hline & $\begin{array}{c}\text { T2, T4, T6 } \\
\text { not optimized }\end{array}$ & $\begin{array}{c}\text { T2, T4, T6 } \\
\text { optimized }\end{array}$ \\
\hline T1pss & 0.1625 & 0.2123 \\
\hline T2pss & 0.0500 & 0.1353 \\
\hline Twpss & 3.0000 & 3.0000 \\
\hline Kpss & 14.5517 & 34.6230 \\
\hline T1svc & 0.1828 & 0.2849 \\
\hline T2svc & 0.3000 & 0.8458 \\
\hline T3svc & 0.1391 & 0.3890 \\
\hline T4svc & 0.3000 & 0.1011 \\
\hline Twsvc & 3.0000 & 3.0000 \\
\hline Ksvc & 278.8829 & 208.5565 \\
\hline
\end{tabular}

The area under curve of $\Delta \omega$ and $\Delta \delta$ for different objective functions are shown in Fig. 7. Employing the objective function $\mathrm{J} 3$ in the optimization problem gives the best response as well as the optimal value for both $\Delta \omega$ and $\Delta \delta$, simultaneously.
The critical clearing time (CCT) is computed for different controllers. The CCT has a small value in case of using the SVC-based stabilizer individually and has a large value when using the SVC in coordinate with the PSS, as shown in Fig. (8).
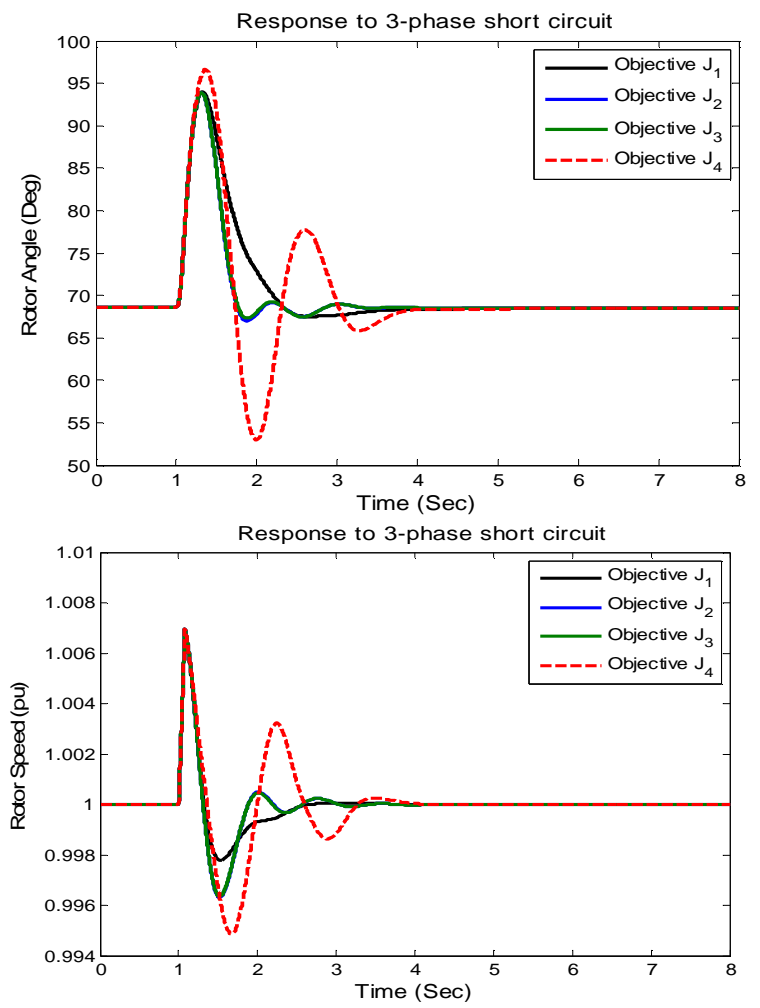

Fig. 6. System response for different objective
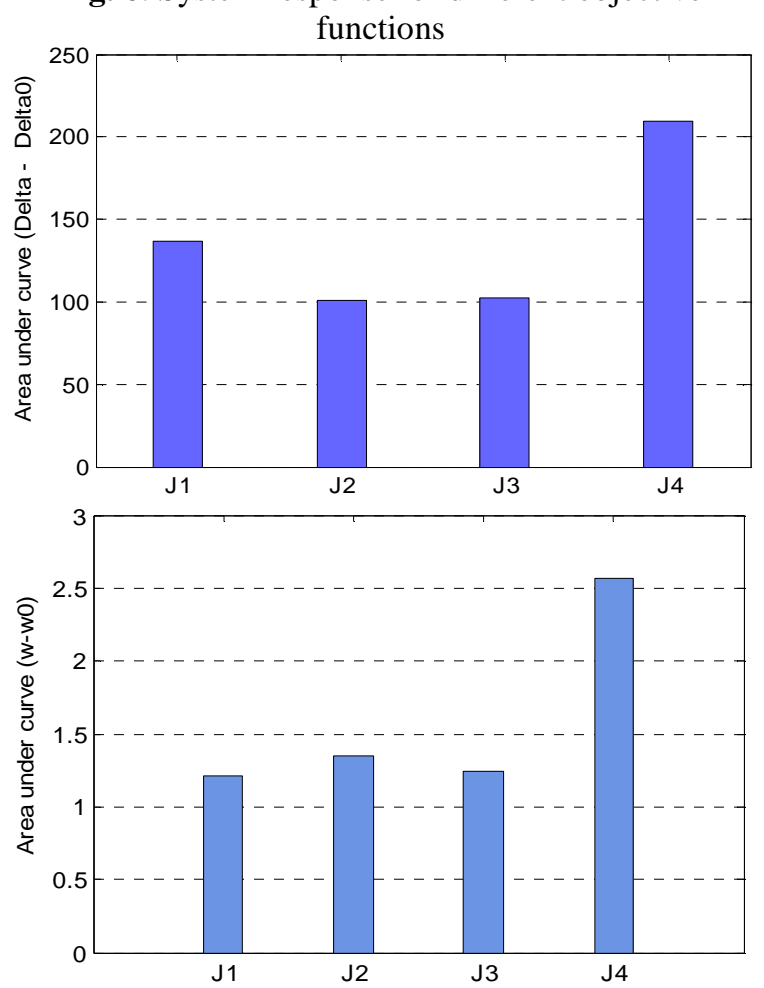

Fig. 7. The area under curve of $\Delta \omega$ and $\Delta \delta$ for different objective functions 


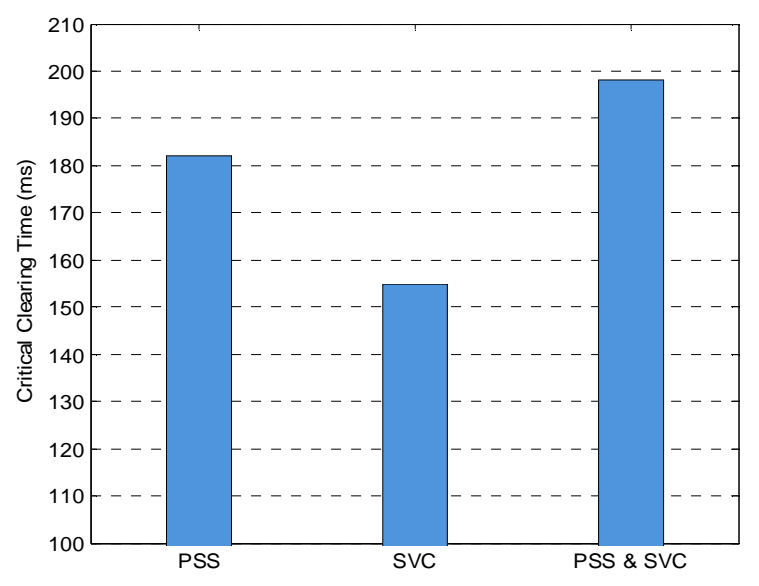

Fig. 8. Critical clearing time for different types of controllers

\section{CONCLUSION}

This paper uses a proposed PSO technique to get the optimal setting of the controllers' parameters. Different objective functions, which are based on the nonlinear model of the system, have been achieved. PSO has approved its ability to coordinate between the PSS and the SVC-based stabilizer. The performance of the system has been enhanced by employing the SVC-based stabilizer with PSS, simultaneously.

More flexibility to the system has been produced due to optimizing all parameters of PSS and SVC controllers. Also, the objective function J3 has been recommended for the optimization process as it gives better response of the system.

Finally, the critical clearing time is increased in case of employing the SVC-based stabilizer in coordinate with the PSS.

\section{REFERENCES}

[1] U. P. Mhaskar, A. M. Kulkarni, "Power Oscillation Damping using FACTS Devices: Modal Controllability, Observability in Local Signals, and Location of Transfer Function Zeros", IEEE Transactions on Power Systems, Vol. 21, No. 1, February 2006, pp.285-294.

[2] Q. Zhao and J. Jiang, "Robust SVC Controller Design for Improving Power System Damping", IEEE Transaction Energy Conversion, Vol. 10, No. 2, Nov. 1995, pp.201-209.

[3] M. H. Haque, "Improvement of First Swing Stability Limit by Utilizing Full Benefit of Shunt FACTS Devices", IEEE Transactions on Power Systems, Vol. 19, No. 4, November 2004, pp. 1894-1902.

[4] Y. L. Abdel-Magid, M. A. Abido, "Robust Coordinated Design of Excitation and TCSCbased Stabilizers using Genetic Algorithm", Electric Power System Research, 69 (2004), pp. 129-141.
[5] M. A. Abido, Y. L. Abdel-Magid, "Coordinated Design of a PSS and an SVC-based Controller to Enhance Power System Stability", Electrical power and energy systems, 25 (2003), pp. 695704.

[6] E. Z. Zhou, "Application of Static VAR Controller to Increase Power System Damping", IEEE Transactions on Power Systems, Vol. 8, No. 2, May 1993, pp.655-661.

[7] M. M. Farasangi, H. Nezamabadi-pour, Y. H. Song, and K. Y. Lee, "Placement of SVCs and selection of Stabilizing signals in Power Systems", IEEE Transaction on power systems, Vol. 22, No. 3, August 2007, pp. 1061-1071.

[8] Yakout Mansour, Wilsun $\mathrm{Xu}$, Fernando Alvarado, Chhewang Rinzin, "SVC Placement using Critical Modes of Voltage Instability", IEEE Transactions on Power Systems, Vol. 9, No. 2, May 1994, pp. 757-763.

[9] J. Baskaran, V. Palanisamy, "Optimal Location of FACTS Devices in a Power System Solved by a Hybrid Approach", Nonlinear Analysis, 65 (2006), pp. 2094-2102.

[10] N. Martins and L. T. G. Lima, "Determination of Suitable Locations for Power System Stabilizers and Static VAR Compensators for Damping Electromechanical Oscillations in Large Scale Power Systems", IEEE Transactions on Power Systems, Vol. 5, No. 4, November 1990, pp.1455-1469.

[11] S. Lee and C. C. Liu, "An Output Feedback Static VAR Controller for the Damping of Generator Oscillations", Electric Power Systems Research, Vol. 25, No. 1, February 1994, pp.916.

[12] Enrique Acha, Claudio R. Fuerte-Esquivel, Hugo Ambriz-Pe'rez, and Ce'sar AngelesCamacho, "FACTS Modeling and Simulation in Power Networks", John Wiley \& Sons Ltd, England, 2004.

[13] Xiao-Ping Zhang, Christian Rehtanz, and Bikash Pal, "Flexible AC Transmission Systems: Modeling and Control", Springer-Verlag Berlin Heidelberg, 2006.

[14] Zwe-Lee Gaing, "Particle Swarm Optimization to Solving the Economic Dispatch Considering the Generator Constraints", IEEE Transactions on Power Systems, Vol. 18, No. 3, August 2003, pp. 1187-1195.

[15] A. A. Abou El-Ela, M. A. Bishr, R. A. F. Saleh and T. Fetouh, "Power systems operation using particle swarm optimization technique", Electric Power Systems Research 78 (2008), pp. 19061913. 